\title{
Honey and Radiation-Induced Stomatitis in Patients With Head and Neck Cancer
}

\author{
Fatemeh Bahramnezhad ${ }^{1}$; Nahid Dehghan Nayeri ${ }^{2}$; Shiva Sadat Bassampour ${ }^{3}$; Mahboobeh \\ Khajeh $^{4,}$; Parvaneh Asgari ${ }^{5}$ \\ ${ }^{1}$ Department of Critical Care Nursing, School of Nursing and Midwifery, Tehran University of Medical Sciences, Tehran, IR Iran \\ ${ }^{2}$ Nursing and Midwifery Care Research Center, School of Nursing and Midwifery, Tehran University of Medical Sciences, Tehran, IR Iran \\ 3 Department of Medical and Surgical Nursing, Faculty of Nursing and Midwifery, Tehran University of Medical Sciences, Tehran, IR Iran \\ 4 Department of Pediatric, School of Nursing and Midwifery, Tehran University of Medical Sciences, Tehran, IR Iran \\ 5 Department of Pediatric, School of Nursing and Midwifery, Tehran University of Medical Sciences, Tehran, IR Iran \\ ${ }^{*}$ Corresponding Author: Mahboobeh Khajeh, Department of Pediatric, School of Nursing and Midwifery, Tehran University of Medical Sciences, Tehran, IR Iran. Tel: +98-2166927171, \\ Fax:+98-2166904252, E-mail:m_khajeh@gmail.com
}

Received: April 2, 2014; Revised: December 2, 2014; Accepted: March 20, 2015

\begin{abstract}
Background: Stomatitis is a common oral complication which affects $100 \%$ of patients undergoing head and neck radiotherapy. Acute stomatitis might cause failure and delay radiotherapy. Attention to mouth hygiene, particularly using mouthwash, has a fundamental importance for these patients.

Objectives: The current study came to addresses the effects of pure natural honey on radiation-induced stomatitis in patients with a variety of head and neck cancers.

Patients and Methods: The present single-blinded nonrandomized controlled trial was conducted on 105 patients undergoing radiotherapy due to head and neck cancer at the radiation unit of Shafa hospital in Kerman, Iran, from October 2012 to March 2012. The research groups were selected by writing the names of the protocols (the mouthwashes of chamomile, honey and the common caring protocol at ward which uses water) on three cubes. The first extracted cube was related to the chamomile mouthwash (Matrica), the second to the honey mouthwash and the last cube to the water mouthwash. The first experimental group $(n=35)$ gurgled a solution containing $20 \mathrm{~mL}$ diluted honey, the second group gurgled a solution containing German chamomile, and the 35 patients in the control group were advised to gurgle $20 \mathrm{~mL}$ water (the ward routine).

Results: The results showed that severe stomatitis in groups of honey, chamomile and control was $0,5.7 \%$, and 17.6\%, respectively. On the 14 th day, it was 0,0 , and $17.6 \%$, respectively. There were significant differences between the three groups regarding the severity of stomatitis in the 14th day $(\mathrm{P}<0.001)$.

Conclusions: The application of natural honey is effective in managing and preventing radiation-induced stomatitis in patients with head and neck cancers.
\end{abstract}

Keywords: Head and Neck Neoplasm; Honey; Mouth; Stomatitis; Radiotherapy

\section{Background}

Among different kinds of malignancies, head and neck cancers are increasing (1). Totally, $4 \%$ of all cancer types have been related to head and neck malignancies $(2,3)$. There are different approaches for treating cancer. The therapeutic methods for treating head and neck cancers including chemotherapy, radiotherapy and surgery affect the individual's life with regard to physical, psychological, social and functional aspects (4). In radiotherapy, the growth and reproduction of cells are thwarted by ionized radiation to the tumor cells, which accordingly destroys them, but has certain side effects (5). Generally, all the patients who undergo the treatment of head and neck via radiotherapy often have oral complications (6). Stomatitis is a common oral complication which affects $100 \%$ of the patients undergoing head and neck radiotherapy (7). Most patients with stomatitis have a set of syacmptoms in- cluding pain, bleeding, wound infection, mouth dryness, change in taste and nutritional situation, and their oral health is also affected. Since mouth has an important role in speaking, receiving nutritional substances and nonverbal communication (smiling, kissing, etc.), a change in its mucous sometimes causes noncompensable complications like a change in the form of mouth, septicemia and death (8). Besides, one third of patients with stomatitis require hospitalization and two-third of them need longterm continuous care after discharge (9). Acute stomatitis might cause failure and delayed radiotherapy (10).

Attention to the mouth hygiene, particularly using mouthwash, has a fundamental importance for these patients. Among the mouthwashes, the ones containing normal saline, ice pieces, honey, allopurinol, benzydamine, chlorhexidine, chamomile and glutamine are

Copyright (c) 2015, Iranian Red Crescent Medical Journal. This is an open-access article distributed under the terms of the Creative Commons Attribution-NonCommercial 4.0 International License (http://creativecommons.org/licenses/by-nc/4.0/) which permits copy and redistribute the material just in noncommercial usages, provided the original work is properly cited. 
worthy of mentioning (11). Nonchemical mouthwashes of honey and chamomile are of these mouthwashes.

Honey leads to preservation of cellular epithelium tissue and prevents the inter-cellular rupture (12). It has been concluded recently that the antiulcer activity of honey is the reason for its antioxidant feature (13). Honey is a natural antibiotic which results in the reduction of the growth of bacteria and prevention of wound infection. Moreover, it prevents the occurrence of wound and leads to its improvement (14). It further can prevent stomatitis (15). Chamomile mouthwash is a nonchemical composition which can prevent the occurrence of stomatitis (16). Chamomile helps the preservation of the cellular tissue and the rupture of cellular tissue is reduced in its presence. Researchers have shown that the antioxidant feature of chamomile helps to preserve the tissue integrity (17). Using chamomile as a mouthwash not only improves the cell wall and prevents rupture, but also helps the patients psychologically and encourages them to keep on with attending to the oral hygiene (18). In addition, the chamomile extract has antibacterial, antiviral and antifungal features (19). In addition to what has been mentioned about the significance of the issue, by using the previous work experience at the radiotherapy ward, the researcher noted that all the patients undergoing head and neck radiotherapy had stomatitis. This complication leads to failure in disease therapy, lack of the individual's interest in continuing the therapy and hospitalization, which would incur enormous expenses on the individuals, their families and the health system.

\section{Objectives}

This work aimed to comparatively study the effects of the two mouthwashes of honey and chamomile in preventing stomatitis among the patients undergoing radiotherapy of head and neck.

\section{Patients and Methods}

The present study was a clinical trial with 105 subjects from October 2012 to March 2012. The sample size was determined by the following formula, $\mathrm{CI}=95 \%$ and power of $80 \%$ (in each group $n=35$ ) (Equation 1 ).

$$
n=\frac{Z_{1-\frac{\alpha}{2}} \sqrt{2 p q}+Z_{1-\beta} \sqrt{p_{1} q+p_{2} q}}{\left(P_{1}-P_{2}\right)^{2}}
$$

To collect data, the researcher referred to the radiotherapy ward of Shafa hospital (Kerman, Iran) after gaining permission from the department of nursing and midwifery of Tehran University of medical sciences and the research deputy of Kerman University of medical sciences. The research groups were selected by writing the names of the protocols (the mouthwashes of chamomile, honey and the common caring protocol at ward, which was water) on three cubes. The first extracted cube was related to the chamomile mouthwash (Matrica), the second to the honey mouthwash, and the last cube to the water mouthwash. To prevent the contact of samples, the researcher filled out all the samples of this protocol initially and after the end of intervention in this group, the recipients of honey were entered into the intervention procedure. After finishing the intervention in this group, the patients in the third group (the control group) were entered to the study. Thereafter, the researcher selected the samples through simple sampling method and the continuous approach, from the patients undergoing head and neck radiotherapy referred to the radiotherapy center of Shafa hospital who had the inclusion criteria (the inclusion and exclusion criteria are presented in Box 1). The first researched group consisted of the patients who received the Matrica mouthwash (chamomile).

Box 1. Inclusion and Exclusion Criteria ${ }^{a}$

\begin{tabular}{l}
\hline Inclusion Criteria \\
\hline Participation to head and neck radiotherapy (nasopharynx, mouth, \\
parotid, neck, sinuses, glossa cancer) \\
Willing to participate \\
Age over 8 years \\
Not treated with steroids \\
Avoidance of tobacco, alcohol, smoking and opiate substances \\
No certain systemic conditions such as diabetes, defect in the immune \\
system, renal disease, liver disease, gastrointestinal disease, asthma or \\
allergic \\
External beam radiotherapy with the $180-200$ cGY dose daily \\
No leucopenia (WBC $<4000 \mathrm{~mm}^{3}$ ) \\
No leukocytosis (WBC $>12000 \mathrm{~mm}^{3}$ ) \\
No thrombocytopenia (platelet count $<100000 / \mathrm{mm}^{3}$ ) \\
Able to gargle mouthwash \\
Able to fully record a form \\
Exclusion Criteria \\
Allergic to the mouth wash \\
Body temperature $>38.5^{\circ} \mathrm{C}$ \\
Nausea and vomiting \\
\hline a Abbreviation: WBC, white blood cell.
\end{tabular}

Before the onset of intervention, the researcher gave the research subjects educational brochures about mouth care methods and provided them with face-to-face instruction about the approach of mouth caring and using mouthwash. They were also given appropriate toothbrushes and toothpastes. On average, prior to the onset of radiotherapy, each patient was given 30 minutes of training and then the feedback was obtained. Moreover, due to the possibility of anxiety in patients, before the unset of radiotherapy and causing disturbance in their learning. During the education, one of the main family members who had an important role in the patient's therapeutic program was also present and the necessary explanations were given to him/ her. The subjects brushed their teeth after each meal and then used mouthwash during three weeks.

The patients receiving the Matrica mouthwash (chamomile) mixed $3 \mathrm{~mL}$ of Matrica drop or chamomile (made 
by Barij Pharmaceutical Company, Iran) with a half-glass water, gargled for 60 seconds and then poured it out of their mouths. If the patients were at the receiving protocol of the honey mouthwash, $20 \mathrm{~mL}$ or five spoonful of honey (honey without wax, produced in Garmsar County spring, Iran, and bought wholesale) was mixed in one glass of water and divided into two equal parts; then, each part was held in mouth for 30 seconds (totally 60 seconds), which was poured out afterwards.

At the common recipient protocol of the ward, the patients used water as their mouthwash after each meal according to the ward routine. However, the frequency and the length of common care at the ward were recorded according to the registration sheet. Besides, this sheet was given to the patients at the two other groups for examining the individuals' observance of the trained cases at the brochure and mouthwash application. Thereafter, they were requested to mark the relevant sheet with an $\left(_{X}\right)$ after brushing their teeth and using mouthwash, after consuming the mentioned food commodities on a daily basis.

The same manner was adopted for dealing with the patients at the common mouthwash receiving group at the ward (water) as the two other groups, so that the researcher provided them with the instructional pamphlet and toothbrush and contacted them every other day to remind them of using the mouthwash.
To examine the patients' mouth with regard to stomatitis, the research colleague examined the condition of stomatitis among the patients and recorded the cases without prior notice about the group of patients before radiotherapy, on days 1, 7 and 14, using the world health organization's (WHO) standard instrument (0 without stomatitis and 4 the severest case of stomatitis with mucous bleeding). Before starting the work, the research colleague was trained by the researcher and the physician about using the checklist for recording the observations produced by WHO and detecting stomatitis for 14 days. Data analysis was performed using SPSS software version 16, with Chi-squared test, Fisher's exact test, Mann-Whitney U, and Friedman test. The Iran registry clinical code (IRCT) was $138902053788 \mathrm{N1}$; the Australian/New Zealand clinical trial registry (ANZCTR): ACTRN12610000847011; endorsement of the ethics committee: /130/6289.

\section{Results}

A total of 104 patients were enrolled until the end of the study (Figure 1). Baseline characteristics and laboratory parameters of the study patients had no statistically significant difference between the groups in terms of gender and age allocation, marital status, education level and history of smoking, as well as basic laboratory parameters (Table 1).

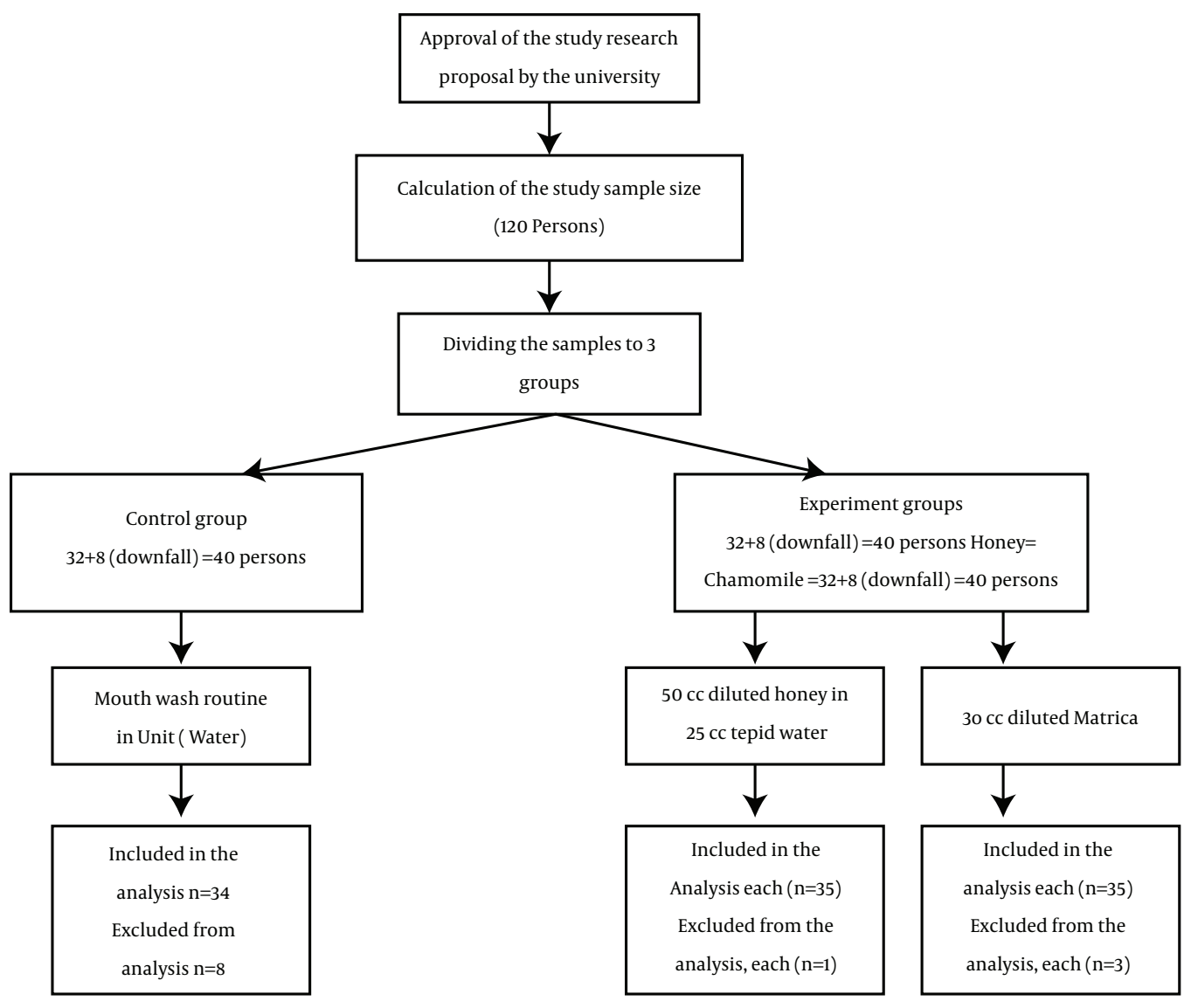

Figure 1. Diagram Plan 
There were no significant differences in tumor staging and the duration of cancer diagnosis between the study groups. The majority of patients in the three groups did not use daily routine tooth brush, tooth stick, tooth pick and mouth washing (Table 2). Before the intervention, alternative tooth brushing was observed only in $17.1 \%$ of the group who administered chamomile, $25.7 \%$ of the patients who administered honey and $17.6 \%$ of the control subjects.

Stomatitis was more severe in both the chamomile and control groups compared with the honey group at days 7 and 14 of assessment (Table 3). In addition, the most severe stomatitis was found in the subjects using only water for mouth washing. However, no significant differ- ence was discovered in the intensity of stomatitis grading between the two experimental groups and the control group in the first day of assessment. Assessment of the tendency of stomatitis grading within the first two weeks of the research protocol showed that the severity of stomatitis in the honey group particularly reduced within the three-week study period, while its severity steadily increased in the control group. In addition, the results showed no significant differences between the three groups regarding the severity of stomatitis in the first day. However, in days 7 and 14 after the intervention, significant differences between the three groups were observed regarding the severity of stomatitis $(\mathrm{P}=0.028$ and $\mathrm{P}=0.00$, respectively).

\begin{tabular}{|c|c|c|c|c|}
\hline Characteristics & Chamomile $(n=35)$ & Honey $(\mathbf{n}=35)$ & Control $(n=35)$ & P Value \\
\hline Male, gender & $22(62.9)$ & $30(85.7)$ & $22(64.7)$ & 0.065 \\
\hline Age, $y$ & $49.6 \pm 17.4$ & $52.0 \pm 17.5$ & $47.8 \pm 19.6$ & 0.630 \\
\hline Married & $28(80.0)$ & $31(88.6)$ & $27(79.4)$ & 0.601 \\
\hline Education level & & & & 0.191 \\
\hline Illiterate & $17(48.6)$ & $12(34.3)$ & $18(52.9)$ & \\
\hline Propa & $3(8.6)$ & $10(28.6)$ & $2(5.9)$ & \\
\hline Elementary & $2(5.7)$ & $4(11.4)$ & $1(2.9)$ & \\
\hline Guidance & $6(17.1)$ & $3(8.6)$ & $8(23.5)$ & \\
\hline High school & $5(14.3)$ & $4(11.4)$ & $3(8.8)$ & \\
\hline College degree & $2(5.7)$ & $2(5.7)$ & $2(5.9)$ & \\
\hline Cigarette smoking & $8(22.9)$ & $5(14.3)$ & $6(17.6)$ & 0.646 \\
\hline \multicolumn{5}{|l|}{ Laboratory parameters } \\
\hline White blood cell $\left({ }^{*} 10^{3}\right)$ & $7.4 \pm 6.1$ & $6.5 \pm 2.0$ & $6.2 \pm 2.3$ & 0.416 \\
\hline Red blood cell & $3.8 \pm 0.7$ & $3.5 \pm 0.7$ & $3.6 \pm 0.6$ & 0.084 \\
\hline Platelet $\left({ }^{*} 10^{3}\right)$ & $157.4 \pm 6.2$ & $144.7 \pm 57.3$ & $134.6 \pm 52.1$ & 0.378 \\
\hline PTT & $29.1 \pm 6.2$ & $31.3 \pm 6.0$ & $31.8 \pm 6.5$ & 0.176 \\
\hline PT & $14.8 \pm 4.9$ & $15.1 \pm 4.9$ & $14.2 \pm 4.0$ & 0.727 \\
\hline INR & $1.4 \pm 0.3$ & $15.1 \pm 0.3$ & $1.3 \pm 0.3$ & 0.509 \\
\hline Cancer & & & & 0.191 \\
\hline Oral cavity & $8(22.9)$ & $10(28.6)$ & $9(26.5)$ & \\
\hline Larynx & $8(22.9)$ & $9(25.7)$ & $7(20.6)$ & \\
\hline Hyperpharynx & $6(17.1)$ & $2(5.7)$ & $4(11.8)$ & \\
\hline Sinus & $3(8.6)$ & $6(17.1)$ & $2(5.9)$ & \\
\hline Gluts & $4(11.4)$ & $2(5.7)$ & $5(14.7)$ & \\
\hline Nasopharynx & $2(5.7)$ & $2(5.7)$ & $3(8.8)$ & \\
\hline Parotid gland & $4(11.4)$ & $4(11.4)$ & $0(0.0)$ & \\
\hline
\end{tabular}

a Abbreviations: PT, Prothrombin time; PTT, partial thromboplastin time; INR, international normalized ratio.

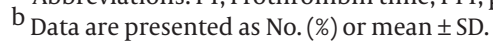


Bahramnezhad F et al.

Table 2. Methods of Tooth Washing in the Study Groups ${ }^{\text {a }}$

\begin{tabular}{|c|c|c|c|c|}
\hline Characteristics & Chamomile $(n=35)$ & Honey $(n=35)$ & Control $(n=35)$ & PValue \\
\hline Toothbrush & & & & 0.095 \\
\hline Never & $13(37.1)$ & $19(54.3)$ & $13(38.2)$ & \\
\hline Seldom & $3(8.6)$ & $5(14.3)$ & $4(11.4)$ & \\
\hline Sometimes & $13(37.1)$ & $2(5.7)$ & $11(32.4)$ & \\
\hline Always & $6(17.1)$ & $9(25.7)$ & $6(17.6)$ & \\
\hline Times of daily brush & & & & 0.905 \\
\hline Once & $18(81.8)$ & $11(78.6)$ & $16(80.0)$ & \\
\hline Twice & $3(13.6)$ & $3(21.4)$ & $3(15.0)$ & \\
\hline Three times & $1(4.5)$ & $0(0.0)$ & $1(5.0)$ & \\
\hline Tooth stick & & & & 0.470 \\
\hline Never & $32(91.4)$ & $32(91.4)$ & $31(91.2)$ & \\
\hline Seldom & $1(2.9)$ & $0(0.0)$ & $0(0.0)$ & \\
\hline Sometimes & $1(2.9)$ & $31(8.6)$ & $1(2.9)$ & \\
\hline Always & $1(2.9)$ & $0(0.0)$ & $2(5.9)$ & \\
\hline Toothpick & & & & 0.879 \\
\hline Never & $30(85.7)$ & $30(85.7)$ & $31(91.2)$ & \\
\hline Seldom & $2(5.7)$ & $3(8.6)$ & $1(2.9)$ & \\
\hline Sometimes & $2(5.7)$ & $2(5.7)$ & $1(2.9)$ & \\
\hline Always & $1(2.9)$ & $0(0.0)$ & $1(2.9)$ & \\
\hline Mouthwash & & & & 0.239 \\
\hline Never & $34(97.1)$ & $33(94.3)$ & $33(97.1)$ & \\
\hline Seldom & $1(2.9)$ & $0(0.0)$ & $0(0.0)$ & \\
\hline Sometimes & $0(0.0)$ & $2(5.7)$ & $0(0.0)$ & \\
\hline Always & $0(0.0)$ & $0(0.0)$ & $1(2.9)$ & \\
\hline Pontic use & $7(20.0)$ & $15(42.9)$ & $11(32.4)$ & 0.121 \\
\hline Duration of pontic use & $10.7 \pm 7.9$ & $11.5 \pm 6.0$ & $12.5 \pm 10.9$ & 0.903 \\
\hline Pontic-related injury & $2(33.3)$ & $3(20.0)$ & $1(10.0)$ & 0.518 \\
\hline
\end{tabular}

${ }^{\mathrm{a}}$ Data are presented as No. (\%) or mean \pm SD.

Table 3. Severity of Stomatitis in the Study Groups ${ }^{\text {a }}$

\begin{tabular}{|c|c|c|c|c|}
\hline Characteristics & Chamomile $(n=35)$ & Honey $(n=35)$ & Control $(n=35)$ & PValue \\
\hline Day 1 & & & & 0.197 \\
\hline None & $7(20.0)$ & $13(37.1)$ & $8(23.5)$ & \\
\hline Mild & $15(42.9)$ & $15(42.9)$ & $10(29.4)$ & \\
\hline Moderate & $13(37.1)$ & $5(14.3)$ & $9(26.5)$ & \\
\hline Severe & $0(0.0)$ & $2(5.7)$ & $6(17.6)$ & \\
\hline Minatory & $0(0.0)$ & $0(0.0)$ & $1(2.9)$ & \\
\hline Day 7 & & & & $<0.001$ \\
\hline None & $11(31.4)$ & $22(62.9)$ & $4(11.8)$ & \\
\hline Mild & $20(57.1)$ & $7(20.0)$ & $10(29.4)$ & \\
\hline Moderate & $4(11.4)$ & $5(14.3)$ & $10(29.4)$ & \\
\hline Severe & $0(0.0)$ & $1(2.9)$ & $8(23.5)$ & \\
\hline Minatory & $0(0.0)$ & $0(0.0)$ & $2(5.9)$ & \\
\hline Day 14 & & & & $<0.001$ \\
\hline None & $17(48.6)$ & $24(68.6)$ & $4(11.8)$ & \\
\hline Mild & $17(48.6)$ & $8(22.9)$ & $11(32.4)$ & \\
\hline Moderate & $1(2.8)$ & $3(8.6)$ & $11(32.4)$ & \\
\hline Severe & $0(0.0)$ & $0(0.0)$ & $6(17.6)$ & \\
\hline Minatory & $0(0.0)$ & $0(0.0)$ & $2(5.9)$ & \\
\hline
\end{tabular}




\section{Discussion}

Oral stomatitis is almost the most common complication of radiotherapy. It could increase the morbidity and mortality rates in patients with head and neck cancer. Hence, it is important to prevent and cure these side effects right after their unset. Oral hygiene and use of suitable mouthwash are among the basic recommendations. Following the administration of honey solution compared with the chamomile and water alone, we discovered that the grade of stomatitis was noticeably condensed within 7 and 14 days of follow-up study, while the patients who used chamomile experienced gradual decrease in stomatitis grading. Nowadays, honey is commonly used for treating infected surgical ulceration, burn wounds and even inflamed tissues. Honey has hygroscopic properties and acidic $\mathrm{pH}$, as well as enzymes, tissue nutrition minerals and vitamins $(15,20)$. Anti-inflammatory effects of honey have been comprehensively studied. In tissue inflammation, honey may reduce edema and pain. Additionally, its phenolic compounds are concerned in inhibitory activities (21). There was significant reduction in symptomatic grades 3 and 4 stomatitis in the honey group and the use of natural honey was effective in prevention and treatment on radiation-induced stomatitis (22).

Fukuda et al. demonstrated that neutrophils induced by honey possessed potent antitumor activity and the effective immune factor of honey was a substrate with a molecular weight of $261 \mathrm{kDa}$ (23). Furthermore, the effect of honey on inflammatory processes might in part be associated with stimulating some inflammatory cytokines (such as TNF- $\alpha$, IL-1 $\beta$ and IL-6) from monocyte cells, which have significant roles in healing and tissue recovery. Another important point is that the observed effects of honey on cytokine production may not be seen in all types of honeys (24). Abdulrahman et al. stated that honey reduced the severity of stomatitis in patients undergoing chemotherapy $(\mathrm{P}<0.05)(25)$.

The present research had the following limitation: It was possible for the project colleague to be unaware of the mouthwash type; however, he was compelled to refer to the patients' houses to examine them for stomatitis by virtue; hence, he could ask them about the type of mouthwash or instead the patients could ask him some questions, so that some clues would emerge that could cause awareness for him, which was out of the researcher's control. Although there was no statistically significant difference between the two types of honey and chamomile mouthwashes in preventing the stomatitis caused by head and neck radiotherapy at the 7th and 14th days after receiving the mouthwashes, the findings indicated that the prevention and improvement trend of stomatitis was better among the patients receiving the honey mouthwash than those receiving the chamomile mouthwash.

Overall, the beneficial effects of honey on stomatitis seemed to be remarkably higher than that confirmed in other appropriate agents and solutions such as chamomile. It appears that honey has a suitable quality for improving inflammation and its use for a two-week period is suitable to repair radiation-induced stomatitis. However, additional studies on the isolation and characterization of these agents are necessary to further clarify the related mechanisms. On the other hand, due to its powerful antitumor effects, honey can prevent the development and growth of tumor, especially for cancers of the mouth area and strengthen the immune system.

The research subjects who were studied by application of the honey mouthwash felt calm during the research and expressed that using honey had led to the relief of pain in their mouth and that they felt better by pouring honey into their mouth. This issue indicates the significance of the fact that honey can be used both as a food substance and at the same time as a mouthwash.

\section{Acknowledgements}

The authors are grateful to the vice-president of research of nursing and midwifery Faculty of Tehran University of medical sciences, Kerman University of Medical Sciences and the patients in the study.

\section{Authors' Contributions}

Study conception design, data collection and analysis, drafting of the document and supervision: Fatemeh Bahramnezhad and Mahboobeh Khajeh. Data collection and drafting of the document: Shiva Sadat Bassampour, Nahid Dehghan Nayeri and Parvaneh Asgari. Drafting of the manuscript: Mahboobeh Khajeh and Fatemeh Bahramnezhad. Critical revision of the manuscript for important intellectual content: Fatemeh Bahramnezhad, Mahboobeh Khajeh, Shivasadat Bassampour, Nahid Dehghan Nayeri and Parvaneh Asgari. Statistical analysis: Fatemeh Bahramnezhad and Parvaneh Asgari.

\section{Funding/Support}

This research was supported by the Research Deputy of Tehran University of Medical Sciences.

\section{References}

1. Russo G, Haddad R, Posner M, Machtay M. Radiation treatment breaks and ulcerative mucositis in head and neck cancer. Oncologist. 2008;13(8):886-98.

2. Vissink A, Jansma J, Spijkervet FK, Burlage FR, Coppes RP. Oral sequelae of head and neck radiotherapy. Crit Rev Oral Biol Med. 2003;14(3):199-212.

3. Ades T, Hinds P, Pierce M. A cancer source book for Nurses. Boston: Jones and Bartlett publishers; 2004.

4. Pandey M, Devi N, Thomas BC, Kumar SV, Krishnan R, Ramdas K. Distress overlaps with anxiety and depression in patients with head and neck cancer. Psychooncology. 2007;16(6):582-6.

5. Suemaru K, Cui R, Li B, Watanabe S, Okihara K, Hashimoto K, et al. Topical application of royal jelly has a healing effect for 5-fluorouracil-induced experimental oral mucositis in hamsters. Meth ods Find Exp Clin Pharmacol. 2008;30(2):103-6.

6. Stokman MA, Spijkervet FKL, Burlage FR, Dijkstra PU, Manson WL, de Vries EGE, et al. Oral mucositis and selective elimination 
of oral flora in head and neck cancer patients receiving radiotherapy: a double-blind randomised clinical trial. Br J Cancer. 2003;88(7):1012-6.

7. Eilers J. When the mouth tells us more than it says-the impact of mucositis on quality of life. Oncol Support Care Q. 2004;1(4):31-43.

8. Eilers J, Million R. Prevention and Management of Oral Mucositis in Patients With Cancer. Semin Oncol Nurs. 2007;23(3):201-12.

9. Murphy BA, Ridner S, Wells N, Dietrich M. Quality of life research in head and neck cancer: a review of the current state of the science. Crit Rev Oncol Hematol. 2007;62(3):251-67.

10. Gibson RJ, Bowen JM, Keefe DM. Technological advances in mucositis research: new insights and new issues. Cancer Treat Rev. 2008;34(5):476-82.

11. Pace S. The Evolution of Mouthwash Contemporary Dental Assisting. J Med Sys. 2008;2(34):7-9.

12. Khanal B, Baliga M, Uppal N. Honey and a Mixture of Honey, Beeswax, and Olive Oil-Propolis Extract in Treatment of Chemotherapy-Induced Oral Mucositis: A Randomized Controlled Pilot Study. Pediatr Hematol Oncol. 2010;29(3):285-92.

13. Ghashm AA, Othman NH, Khattak MN, Ismail NM, Saini R. Antiproliferative effect of Tualang honey on oral squamous cell carcinoma and osteosarcoma cell lines. BMC Complement Altern Med. 2010;10(1):49.

14. Segovia D. The Clinical Benefits of Active Leptospermum Honey in Oncologic Wounds. Ostomy Wound Manag. 2010;56(10):10.

15. Biswal BM, Zakaria A, Ahmad NM. Topical application of honey in the management of radiation mucositis: a preliminary study. Support Care Cancer. 2003;11(4):242-8.

16. Mazokopakis EE, Vrentzos GE, Papadakis JA, Babalis DE, Ganotakis ES. Wild chamomile (Matricaria recutita L.) mouthwashes in methotrexate-induced oral mucositis. Phytomedicine.
2005;12(1-2):25-7.

17. Yang ES, Murphy BM, Chung CH, Netterville JL, Burkey BB, Gilbert J, et al. Evolution of clinical trials in head and neck cancer. Crit Rev Oncol Hematol. 2009;71(1):29-42.

18. Tiemann P, Toelg M, Ramos F. Administration of Ratanhia-based herbal oral care products for the prophylaxis of oral mucositis in cancer chemotherapy patients: a clinical trial. Evid Based Complement Alternat Med. 2007;4(3):361-6.

19. Wang Y, Tang H, Nicholson JK, Hylands PJ, Sampson J, Holmes E. A metabonomic strategy for the detection of the metabolic effects of chamomile (Matricaria recutita L.) ingestion. J Agric Food Chem. 2005;53(2):191-6.

20. Bardy J, Molassiotis A, Ryder WD, Mais K, Sykes A, Yap B, et al. A double-blind, placebo-controlled, randomised trial of active manuka honey and standard oral care for radiation-induced oral mucositis. BrJ Oral Maxillofac Surg. 2012;50(3):221-6.

21. Van der Weyden EA. The use of honey for the treatment of two patients with pressure ulcers. Br J Community Nurs. 2003; 8(12):S14-20.

22. Kassim M, Achoui M, Mansor M, Yusoff KM. The inhibitory effects of Gelam honey and its extracts on nitric oxide and prostaglandin E(2) in inflammatory tissues. Fitoterapia. 2010;81(8):1196-201.

23. Maiti PK, Ray A, Mitra TN, Jana U, Bhattacharya J, Ganguly S. The effect of honey on mucositis induced by chemoradiation in head and neck cancer. J Indian Med Assoc. 2012;110(7):453-6.

24. Fukuda M, Kobayashi K, Hirono Y, Miyagawa M, Ishida T, Ejiogu EC, et al. Jungle honey enhances immune function and antitumor activity. Evid Based Complement Altern Med. 2010;6(1):113.

25. Abdulrahman M, Samir Elbarbary N, Ahmed Amin D, Saeid Ebrahi R. Honey stimulates inflammatory cytokine production from monocytes. Cytokine. 2003;21(5):242-7. 